\title{
ECONOMÍA POLÍTICA DEL UNHEIMLICH EN EL PENSAMIENTO (ESTÉTICO-POLÍTICO) DE HEIDEGGER ${ }^{1}$
}

\section{Political economy of the Unheimlich in Heidegger's aesthetic-political thought.}

Luciano Allende Pinto
Universidad de Chile lallende1978@gmail.com

\section{Resumen:}

El presente texto aborda el problema de la política en el pensamiento de Heidegger a partir del tratamiento que hay en él de la cuestión de la verdad y la obra de arte, exponiendo a partir del concepto de Unheimlich, los elementos centrales de una ontología política que problematiza los acercamientos habituales al "caso Heidegger", hacia una consideración filosófica política donde ese problema pueda ser pensado en relación con el concepto de pensar poético rememorante (Andenken).

Palabras clave: Unheimlich, Andenken, ontología política, pensamiento poético.

\footnotetext{
${ }^{1}$ Este texto fue presentado en el Coloquio de Estudiantes de Postgrado "Arte, Política y Estética", organizado por la Facultad de Filosofía y Humanidades de la Universidad de Chile, enero de 2014.

2 Licenciado en Filosofía por la Universidad ARCIS, becario CONICYT en el Programa de Doctorado en Filosofía, mención Estética y Teoría del Arte, de la Universidad de Chile. Docente de la Facultad de Ciencias de la Educación de la Universidad San Sebastián (USS), sede Santiago, en los cursos de "Ética de la Profesión Docente" y "Persona Humana y Sociedad".
} 


\section{Abstract:}

This present paper deals with the problem of politics in Heidegger's thought from the treatment he gives to the question of the truth and the artwork, exposing, from the concept of Unheimlich, the core elements of a political ontology that problematizes the common approaches to the "Heidegger case" towards a political philosophical consideration where that issue can be treated in relation to the concept of a remembering poetic thought (Andenken).

Keywords: Unheimlich, Andenken, political ontology, poetic thought.

Fue con ocasión de la publicación de Holzwege en 1950 que Heidegger definió su propio pensar como camino; un camino que pensado a grandes rasgos va desde el trabajo con la fenomenología hermenéutica, hasta la búsqueda, de un nuevo inicio, en el Ereignis, o bien, va desde la pregunta por el sentido, a través de la pregunta por la verdad, para decantar finalmente en el problema del tópos donde sentido y verdad tienen otro lugar. Los pasos del pensador, le hacen recorrer la historia de lo pensado, y lo dicho, por una tradición cuyo decir olvidaría lo más digno de ser pensado y ocultaría en ese olvido lo esencial; frente a esto, su pensar se arrojaría existencialmente en una búsqueda incesante de lo no pensado en lo pensado, y lo no dicho, en lo dicho, cuestión que lo conducirán al enfrentamiento de su pensar con las propias condiciones de posibilidad e imposibilidad del decir y el pensar, en cuanto tales.

El temprano anuncio de la tarea de la Destruktion en 1927, así como la necesidad de introducir el arcaísmo Seyn, 
en la década del '30 para retrotraer a la escucha las resonancias de un sentido más originario del Ser, así como la tachadura de la palabra misma en la década del ' 50 , son operaciones donde más allá del gesto, lo que se juega es un pensamiento que intenta situarse, en el límite externo y originario de sus propias posibilidades.

Es en dicho trabajo, con el pensar y con el decir expuestos por y en el trabajo de Heidegger, que éste se abre tempranamente a la poesía, a un decir y un pensar poéticos, que al menos por una de sus aristas se expondrá a continuación.

Es necesario señalar en primera instancia que las reflexiones de Heidegger sobre el arte, marcan un punto de inflexión en su pensar, en la medida en que por una parte, ponen efectivamente en juego, el paso desde un pensar que se encuentra atrapado en la lógica del desentrañamiento del sentido, hacia un pensar que se pregunta por el asunto propio de la verdad. Comprendida ésta en tanto articulación y diferencia Alétheia/Lethé o des-encubrimiento de lo velado, es que se abre una noción de verdad, cuyo obrar encuentra en el arte un poder fundacional que no sólo excede toda consideración de la verdad como concordancia o adequatio, sino que sería incluso su fondo.

Nos dice Heidegger en De la esencia de la verdad:

El develamiento del ente como tal es a la vez y en sí la disimulación del ente en totalidad. Es en esta simultaneidad del develamiento y de la disimulación que se afirma el errar. La disimulación de lo obnubilado y el errar pertenecen a la esencia originaria de la verdad [in das anfängliche Wesen der Wahrheit]. La libertad, comprendida a partir de la ex-sistencia insistente del Dasein, no es la esencia de la verdad (como conformidad de la presentación) sino porque la libertad deriva ella misma de la esencia originaria de la verdad, del 
reino del misterio en el errar. El dejar-ser del ente se efectúa mediante nuestro comportamiento en el seno de lo abierto. Sin embargo, el dejar-ser del ente como tal y en totalidad, no se realiza auténticamente más que cuando, de un instante a otro, él es asumido en su esencia originaria. En ese momento, la aceptación resuelta del misterio comienza a cumplirse en el seno del errar percibido como tal. Desde ese momento, la cuestión de la esencia de la verdad se halla planteada en su radical originalidad. A partir de entonces, se devela el origen de la imbricación de la esencia de la verdad con la verdad de la esencia (1952: 21).

Seguido de lo anterior, se considera que los análisis, especialmente desarrollados en El origen de la obra de arte en 1936, romperían aquella reducción del arte a estética y a "mera expresión de la vida interior del hombre", que en su conferencia posterior La época de la imagen del mundo en 1938, se consignará como uno de los elementos constitutivos de la época moderna, época, que el mismo Heidegger quiere "superar".

A esta superación de la noción estrictamente esteticista del arte, sumamos por otra parte, la consignación de que dichas reflexiones avizoran junto a la noción de técnica, un modo de pensar la producción no circunscrito a la indagación óntica sobre el modo de ser del útil, sino que se abre al problema de la fundación de mundo, y al decir poético, cuya primera indicación exige prestar atención a la política que ahí se juega, antes de toda reducción de aquella a ontología regional -siguiendo una nomenclatura propuesta por el propio Heidegger en Ser y Tiempo, y en el recaudo de toda Metapolítica vaciada de consideraciones "existenciales".

Antes de un segundo paso, se hacen necesarias dos aclaraciones preliminares sobre los conceptos que a 
continuación se desarrollarán: "Andenken" y "Ontología política".

Respecto de la noción de Andenken. Con el término Andenken, se quiere significar la comprensión que el propio Heidegger hace de un pensar capaz de superar la tradición metafísica y su pensar calculador, hacia un pensamiento que a la vez que sea capaz de relacionarse con lo incalculable, atisbe bajo la noción de "pensar poético originario", un potencial de fundación mayor a aquel que la filosofía misma posee en su versión ontoteológica. Es decir, aquel no sólo invita a pensar la fundación que pensadores y poetas son capaces de realizar, sino además señala la extensión de los límites del pensar y el decir, hacia el des-velamiento de lo no dicho en lo dicho y lo no pensado en lo pensado.

Tal ejercitación del pensar, como un pensar poético rememorante, busca retrotraer el pensar mismo a una experiencia más originaria, a una experiencia capaz de abrir destinos históricos donde la filiación, esto es, la familiaridad del Ser al hombre pueda ser pensada con mayor profundidad. El pensar poético originario -propio de la poesía pensanteenunciaría eventos originarios que abren la fundación/donación de mundo en una pluralidad de sentidos cuya familiaridad con el Ser en cuanto Hogar al cual regresar - Heimkunft- mienta la constitución de una familiaridad más profunda que cualquier besorgen-vertrauten como ha sido pensada en Ser y Tiempo.

Pensamiento poético rememorante y obra de arte anudan el sentido inaugural que posee la obra de arte. El lenguaje poético, tendría la capacidad de poner en obra la verdad y en ese poner obra, producir la relación del Ser al 
Hombre como fundación de mundo(s), cuestión que no será posible si nos quedamos atrapados en un lenguaje representacional y un pensar de ese orden. Expresiones de esa poesía pensante, serían Hölderlin, Sófocles, y Trakl, entre otros. Para dar cuenta de este asunto revisaremos algunos análisis de Heidegger sobre un pasaje de la obra de Sófocles.

Respecto del problema político en Heidegger, y la posibilidad de comprender los desarrollos de la pregunta por el Ser, como una ontología política, se recoge la observación de Lyotard, acerca de la necesidad de mantenerse en cierta distancia respecto de los modos en que se habría dado en la escena de pensamiento francesa, la recepción concerniente al "caso Heidegger" tras la denuncia de Víctor Farías, a partir de la cual se sucedieron un número importante de publicaciones, que oscilaban entre dos compulsiones extremas de denuncia y silenciamiento. Y junto a ello tener en cuenta la observación del propio Lyotard quien además de rescatar el trabajo de Jacques Derrida, y Phillipe Lacoue-Labarthe, a partir de las nociones de espíritu el primero y de mito(política) y mitopoíesis, en el segundo, como aquellas que habrían pensado el asunto con una profundidad y seriedad adecuadas. Sin caer en las interpretaciones centradas en lo anecdótico y biográfico que se producen bajo el nudo que significa la sola idea de un "expediente Heidegger"; Lyotard da cuenta del caso Heidegger a partir de un "cierto conocimiento" o "incierto conocimiento" -voluntario o involuntario- que se desata a partir del trabajo interpretativo de lo político a partir del poema en Lacoue-Labarthe y la presencia de lo espiritual de parte de Derrida, en tanto se sigue que "el caso se nos presenta en el modo de lo Unheimliche, familiar y extraño" a la 
vez. Dejamos pendiente las consideraciones respecto de la recepción local de la obra de Farías, y los abordajes propuestos por Oyarzún (2001) donde se propone la necesidad de pensar la relación entre filosofía y poder, y el problema de la traducción como destino de la filosofía en Chile.

De momento recogemos de Lyotard la constatación de la emergencia de la denuncia en la escena de discusión francesa, en cuyo comentario atribuye a lo Unheimlich una connotación central: "El 'nazismo' de Heidegger y su silencio, pertenecen a esta misma economía de lo Unheimlich" (JeanFrançois Lyotard, 1988: 59).

La observación de Oyarzún sobre la traducción es fundamental, porque trasciende la idea de un "destino" para el quehacer filosófico local, ya que no se debe pasar por alto que con lo Unheimlich se da también una suerte de traducción interna a la lengua alemana (Hölderlin-Heidegger), y a su vez, el mismo concepto se presenta como un espacio propicio para pensar el problema de lo político desde Heidegger, asumiendo que en su espectral presentarse, lo Unheimlich posee un potencial deconstructor que puede poner en crisis toda fundamentación de mundo, toda polis, por supuesto todo Reich, e incluso todo "caminar", es decir, intuyo que el potencial deconstructor de lo Unheimlich, tiene el poder de poner en crisis, todo ser, construir, habitar, pensar.

No es una cuestión aislada que con posterioridad a Ser y Tiempo, lo Unheimlich reaparezca tras su irrupción en los cruciales $\S \S 41$ y 57 , pero ya no será para ni para exponer con cierta lateralidad el sentido propio del "estar en" del seren-el-mundo, ni para señalar el silencio de fondo que conlleva 
la voz de la conciencia propia del Dasein, sino para indicar el modo de ser del hombre que sería des-encubierto en la tragedia sofoclídea, como expresión ejemplar del pensar poético griego. Es en este sentido, que la cuestión de lo Unheimlich se anuda con la comprensión heideggeriana del arte y su relación con la verdad y el poder de la verdad, en cuanto, verdad del poder.

Sobre la obra de arte. Para Heidegger lo que sea el arte en tanto "la puesta en obra de la verdad", mienta un pólemos (combate) por la apertura de mundo. La obra propiamente tal, cuyo obrar, en tanto, pone en obra la verdad irrumpe en el mundo con fuerza inaugural, tal que cualquier análisis circunscrito al carácter cósico de la obra, no haría sino corresponder al extravío vulgar en lo que de circunmundano tiene la obra. Sería a partir de su potencial poiético, de la producción y un producir no cósico ni intramundano que Heidegger advierte una forma privilegiada de poíesis, en la poesía (Dichtung), y lee en ella el carácter esencial del arte. Entiende que el arte cuyo obrar es el lenguaje sería aquel cuya puesta en obra (poíesis, productio) conllevaría el acontecimiento de la verdad, en la medida en que el pensamiento poético podría o estaría capacitado para el desencubrimiento que la verdad exige para ser tal.

Así entendemos que pensar el arte, tanto como pensar la técnica deben recoger el problema de la producción como producción de un mundo, donde lo construido es una familiaridad constitutiva de modos de conducirse y habitar. Ahora bien, tal inauguración señala la relación Verdad, Arte y Poesía como un mismo fenómeno de apertura: 
La verdad como claro y encubrimiento de lo ente acontece desde el momento en que se poetiza. Todo arte es en su esencia poema en tanto que un dejar acontecer la llegada de la verdad de lo ente como tal. La esencia del arte, en la que residen al tiempo la obra de arte y el artista, es el ponerse a la obra de la verdad. Es desde la esencia poética del arte, desde donde éste procura un lugar abierto en medio de lo ente en cuya apertura todo es diferente a lo acostumbrado (Martin Heidegger, 1998a: 52).

Pero quién, más allá del artista, puede ejercer esa producción, donación, o poetización. Quién puede dar ese combate por la verdad, y en la verdad, en cuando mundos habitables, o bien, como se pregunta Heidegger, ¿Quién es el hombre de ese decir poético? ¿Quién es el que abre el pensamiento y el decir a lo des-acostumbrado? El propio Heidegger da otro indicio de cómo se debe pensar esa diferencia con un habitar familiar:

El poner a la obra de la verdad hace que se abra bruscamente lo inseguro (Un-geheure) y, al mismo tiempo, le da la vuelta a lo seguro y todo lo que pasa por tal. La verdad que se abre en la obra no puede demostrarse ni derivarse a partir de lo que se admitía hasta ahora. La obra rebate la exclusividad de la realidad efectiva de lo admitido hasta ahora. Lo que el arte funda no puede nunca, precisamente por eso, verse contrarrestado por lo ya dado y disponible. La fundación es algo que viene dado por añadidura, un don (1998a: 54).

Tal verdad inseguriza, es una verdad Un-geheure, inmensa, extraordinaria, incluso monstruosa; curiosamente, se trata del mismo término que Heidegger desiste de utilizar, cuando trabaja en el curso Introducción a la metafísica de 1935 el primer canto del coro de Antígona, reemplaza para la expresión griega tó deinón de la traducción que Hölderlin habría propuesto, por el término Unheimlich, que en la versión castellana del curso suena "lo pavoroso". Tal anécdota de traducción nos retrotrae a las consideraciones preliminares 
que hicimos sobre la traducción por una parte y a las que dejamos resonando sobre lo Unheimlich, por otro. Profundizaremos en este último asunto.

Emergencia de lo Unheimlich, en el curso de 1935, lo "pavoroso", será fundamental para no sólo atender la pregunta por el quién es el nosotros, señalado por Heidegger, como se pregunta Derrida, sino "juzgar quién es el hombre en éste decir poético" (Martin Heidegger, 1999: 137).

Atendamos a estos pasajes. Los primeros versos del coro rezan: "Muchas cosas son pavorosas;/ nada, sin embargo, sobrepasa al hombre en pavor". A decir de Heidegger estos dos primeros versos anticipan todo el canto, la interpretación heideggeriana de éste, nos dice: "el hombre es tó deinótaton, lo más pavoroso. Éste decir capta al hombre desde los límites más extremos y desde los escarpados abismos de su ser [...] Sólo a un proyecto poético y pensante se le revela un tal ser" (1999: 137).

Pero ¿qué quiere decir que el hombre sea más pavoroso que aquellas muchas otras cosas? Es decir ¿qué se busca con pensar al hombre como lo más pavoroso de lo pavoroso? ¿Qué puede significar la decisión de Heidegger de traducir tó deinón como Unheimlich, contra la traducción de Hölderlin, como monstruoso [Ungeheueres]?

Nos dice Heidegger que tó deinón tendría esa "pavorosa ambigüedad con la que el decir de los griegos recorría en direcciones opuestas los enfrentamientos con el ser" (1999: 138); así -si se quiere más inmediato- tó deinón mienta lo terrible, como una violencia sometedora, y un imperar, que se dicen de la relación hombre y Physis. 
El deinón es lo terrible en el sentido del imperar que somete [überwältigf], que impone el pánico, la verdadera angustia y también la intimidación concentrada y callada que se agita en sí misma. La violencia, lo que somete, constituye el carácter esencial del imperar mismo. Allí donde irrumpe, puede retener en sí mismo su poder sometedor. Pero no por eso es más inofensivo sino todavía más terrible y más extraño.

En otro sentido, deinón significa violencia, en el sentido de que aquel que la usa no sólo dispone de ella sino que es violento en la medida en que el empleo de la violencia para él no sólo constituye un rasgo fundamental de su conducta sino de toda su existencia (1999:138).

Entender la inseguridad propia de la fundación de mundo como Unheimlich, invita a repensar el pólemos originario de la verdad, abre el problema a una economía de la violencia, donde ésta se presenta en un primer lugar como violencia sometedora, entendida como determinación existencial del hombre, su economía política presenta lo pavoroso ya no como mero rasgo del hombre, sino como la condición según la cual el poder mismo es un asunto propiamente humano. Así nos dice Heidegger:

El ente en su totalidad, en tanto lo que impera, es lo que somete causando pavor, deinón, en el primer sentido. Pero el hombre es deinón, por un lado, cuando permanece expuesto a lo que lo somete ya que pertenece esencialmente al ser y, por otro, es deinón porque es el que hace violencia (más allá del mero acto violento, sino caracterizada desde el ex-sistir, Así) [junta lo que impera y lo deja entrar en un estado manifiesto]. El hombre no hace violencia además y al lado de otras acciones, sino sólo en el sentido de que a causa de su actividad violenta y con ella, usa la violencia contra lo que lo somete (1999: 139) ${ }^{3}$.

${ }^{3}$ Explicitación entre corchetes del propio Heidegger. 
Inmediatamente después de hacer esta interpretación que pone el énfasis en la violencia originaria, o violencia del origen, nos habla Heidegger de la elección del término Unheimlich, ahora con guión:

\begin{abstract}
Entendemos lo pavoroso [Un-heimlich] como aquello que nos arranca de lo familiar [heimlich], es decir, de lo doméstico, habitual, corriente e inofensivo. Lo pavoroso no nos permite permanecer en nuestra propia tierra [einheimlisch]. En esto reside lo sometedor. Pero el hombre es lo más pavoroso no sólo porque su esencia transcurre en medio de lo pavoroso así entendido, sino porque se pone en camino y trasciende los límites que inicialmente y a menudo le son habituales y familiares. Porque él, entendido como el que hace violencia, sobrepasa los límites de lo familiar, siguiendo justamente la dirección de lo pavoroso o no-familiar, entendido como poder sometedor (1999:139).
\end{abstract}

Éste sobrepasar lo familiar propio del hombre es lo que en Ser y Tiempo fue considerado como un arrancar al Dasein de la familiaridad como Vertrautenheit, en tanto revelar el estar-encasa como un no-estar-en-casa, que a partir de la disposición afectiva fundamental de la angustia abría al Dasein a lo que él es en propiedad. Como vemos, casi diez años después de Ser y Tiempo, el término Unheimlich releva el Cuidado [Sorge] como modo fundamental del ser del Dasein, a partir de una determinación ontológico política del hombre. Se juega en dicho cambio terminológico un triple relevo, que va en primer lugar de la pregunta por el sentido a la pregunta por la verdad; aquel cambio en el preguntar, implica necesariamente un desvío en el camino del proyecto, en la medida en que exigirá otra tarea, ya no se tratará de la destrucción y sus hitos anunciados en Ser y Tiempo, sino de la búsqueda de cierta superación (Destruktion/Überwindung), y con ello finalmente, 
el paso (poros) desde una ontología fundamental a una ontología política.

Asumimos que el siglo $\mathrm{XX}$ y las experiencias del fascismo como devastación radical de los cuerpos y el sentido, no serían otra cosa que la expresión de un mundo y un habitar, cuya condición no parece ser otra sino pavorosa.

Para entender en todo su alcance estas palabras del coro acerca del hombre, debemos tener en cuenta que la expresión que caracteriza al hombre como tó deinótaton, lo más pavoroso, no pretende atribuirle una cualidad especial, como si por lo demás fuese otra cosa. Antes bien, esa palabra significa que ser lo más pavoroso constituye el rasgo fundamental de la esencia del hombre, en el cual se inscribirán desde y hasta siempre todos los demás rasgos (Martin Heidegger, 1999: 140)

Pero como indicamos antes el uso del término Unheimlich por parte de Heidegger, junto con instalar la necesidad de pensar una suerte de ontopolítica guarda un potencial deconstructor de toda ontología política por cuanto, la apertura que aquel poder implica, produce una suerte de extravío político fundamental. Esto por cuanto sin salir del análisis propuesto de Antígona, nos señala el texto dos condiciones fundamentales que serían constitutivas del tó deinótaton, a saber, su carácter aporético y apolítico.

Respecto del aporos y el apolis como rasgos fundamentales que constituyen lo Unheimlich y su inhospitalidad constitutiva de nuestro ser-en-el-mundo, atendamos a aquel pasaje del texto trágico y al comentario propuesto por Heidegger. Con especial atención al primero, por cuanto el mismo Heidegger ha definido con ocasión de la publicación de Holzwege su experiencia del pensar como camino. 
'Por todas partes viaja sin cesar: desprovisto de experiencia y sin salidas llega a la nada' Las palabras esenciales son pantoporos y aporos. El vocablo poros significa: a través de..., en tránsito a..., camino. Por todas partes el hombre se abre camino; se atreve a avanzar hacia todos los dominios del ente, del imperar que somete $y$, precisamente al hacerlo, es arrojado fuera de todo camino. Sólo por esta razón comienza a abrirse la plena condición de pavor, propia de lo más pavoroso. El hombre no sólo experimenta el ente en su totalidad como lo pavoroso; siendo él mismo aquel que hace violencia, no sólo va más allá de lo que le es familiar, sino que además llega a ser en todo lo más pavoroso: a él le alcanza la até, la ruina, la desgracia, y en tanto que todos los caminos carecen de salidas, se ve arrojado fuera de cualquier relación con lo familiar (Martin Heidegger, 1999: 140).

Éste ser aporético, éste estar sin salida en el camino, en tanto encaminado, es justamente la condición política que caracteriza tanto el despliegue de la técnica como nuestro modo de ser contemporáneo. El arrojo, no sería entonces sólo caída en el mundo y lo intramundano, sino arrojo fuera de toda familiaridad con el mundo mismo, perplejidad y desazón que vienen con la ruina, y abren el carácter de más pavoroso como modo de ser propio del hombre. Ese encaminarse aporético, habla también de la imposibilidad de no caminar, de no estar en la historia. El paso -poros- y su no-paso aporos- como camino y sin-camino constituyen el serarrojado fuera de lo familiar propio de lo Unheimlich: es éste último aquel concepto que en cuanto sombra de lo incalculable, excede los límites de la representación y del mundo moderno, es aquel que para ser si no ya pensado, al menos "meditado", mienta una parálisis de la reflexión de una propuesta política al modo en que los pensadores de la política en tanto disciplina o incluso como ontología regional 
piensan, es aquel pavor el que arranca de cuajo al pensar sobre el presente hacia la poesía.

¿Qué mundo es el que queda ahí donde el devenir histórico del siglo XX y las primeras décadas de XXI, nos ponen en un extravío fundamental? Quizá sea necesario pensar al hombre y nuestro momento histórico no sólo como aporos sino, como señala el mismo Heidegger, también como apolites, Respecto de esto último citará Heidegger la expresión: upsepolisapolis. os dice el pensador:

Podemos observar que esta expresión [upsepolisapolis] está construida de la misma manera que la anterior pantoporosaporos [...] Sin embargo, se refiere a otra orientación del ente. No dice poros sino polis; no menciona todos los caminos del ámbito del ente, sino el fundamento y el lugar de la existencia del hombre mismo, el lugar de la encrucijada de todos esos caminos: la polis. Polis se traduce por Estado y ciudad; pero estas acepciones no aciertan en su pleno sentido. Antes bien, polis quiere decir el lugar, el allí, dentro del cual y en el cual es el ser-allí, la ec-sistencia entendida como histórica. La polis es el lugar de la historia, el allí en el cual y a partir del cual y para el cual acontece la historia (1999: 140).

En sentido disciplinar es fundamental señalar la necesidad de pensar la historia y la historicidad a partir de la interpelación del ser [An-spruch], que no hará sino concebir una "filosofía de la historia", una política, y un mundo, cuya marca fundamental es la crisis y la ruina, donde una filosofía del fin buscará herramientas en el borde de su propia historicidad: la poesía, aquellos expulsados de la República en la escena platónica fundacional de Occidente, reaparecen como un fantasma, de modo Unheimlich para sugerir un pensar poético que intente mantenerse en aquel "entre" que es fundamento desfondado y fractura, tanto de la modernidad como de la 
existencia misma, su darse siniestro no es otra cosa que el doble filo de lo incalculable propio de aquella "economía de lo Unheimlich". Dicha economía mienta entonces, tanto pensar al hombre como a la verdad, que desde su fondo estético político, ya no sólo funda un Estado (1998a: 45), sino que además es capaz de desmontar toda articulación política. Si esta última economía sin oikos y el arte son el lugar propicio para seguir pensando lo que queda de mundo, es algo imposible de señalar con certeza. Sin embargo, tanto como el fin de la filosofía mienta el inicio de la tarea del pensar, la parálisis política que aquí acontece, en tanto articulación, aporética y apolítica, reclamará la tarea de pensar otras políticas y estéticas; e inaugurar otros conceptos que recojan un desafío tal, pertrechos conceptuales en un combate que se dé, de cara tanto a la historia, como a nuestra aporética cotidianidad.

\section{Bibliografía}

DERRIDA, Jacques. (2001). Del Espíritu. Trad. de Alejandro Madrid Zan. Santiago: Edición electrónica philosophia.cl

FARíAS, Víctor. (1998). Heidegger y el nazismo [1987]. Santiago: Fondo de Cultura Económica.

Farías, Víctor; OYARzún, Pablo y FontaIne, Arturo. (2001). "Heidegger y la Política" [1998]. Revista Centro de Estudios Públicos, 83, 18-43. 
HeidegGer, Martin. (1952). "De la esencia de la verdad" [1930]. Revista Cubana de Filosofía, II, 2, 5-22.

Heidegger, Martin. (1997). Ser y tiempo [1927]. Trad. de J. E. Rivera. Santiago: Edit. Universitaria.

HEIDEGGER, Martin. (1998a). "El origen de la obra de arte" [1936]. En: Caminos de bosque. Madrid: Alianza Editorial, 1162.

HeIDEgGer, Martin. (1998b). "La época de la imagen del mundo" [1938]. En: Caminos de Bosque. Madrid: Alianza Editorial, 63-90.

HEIDEGGER, Martin. (1999). Introducción a la metafísica [1935]. Madrid: Gedisa .

HeidegGer, Martin. (2001). "Carta sobre el «Humanismo»" [1946]. En: Hitos. Madrid: Alianza Editorial, 259-297.

HeIDEgGeR, Martin. (2005a). "Hölderlin y la esencia de la poesía" [1936]. En: Aclaraciones a la poesía de Hölderlin [1936- 1968]. Madrid: Alianza Editorial, 37-54.

HeidegGer, Martin. (2005b). "Memoria (Andenken)" [1943]. En: Aclaraciones a la poesía de Hölderlin. Madrid: Alianza Editorial, 87-168.

HeidegGer, Martin. (2005c). "Regreso al Hogar/A los parientes (Heimkunft)" [1943]. En: Aclaraciones a la poesía de Hölderlin. Madrid: Alianza Editorial, 11-38. 
Heidegger, Martin. (2006). Aportes a la filosofía: Acerca del Evento [1936-1938]. Buenos Aires: Editorial Biblos.

Lacoue-Labarthe, Philippe. (2002). La ficción de lo político [1987]. Madrid: Arena Libros.

LACOUE-LABARTHE, Philippe. (2006). La poesía como experiencia [1997]. Madrid: Arena Libros.

LYOTARD, Jean-François. (1995). Heidegger y los judíos [1988]. Buenos Aires: La marca.

OYARZún, Pablo. (2001). "Heidegger: Tono y Traducción" [1989]. En: De lenguaje, historia y poder. Santiago: Departamentode Teoría de las Artes, 101-127. 